We use cookies to improve our service and to tailor our content and advertising to you. More info $\bigotimes$ Close You can manage your cookie settings via your browser at any time. To learn more about how we use cookies, please see our cookies policy. Close

Intended for healthcare professionals

thebmj

\title{
Editorials
}

\section{Reducing sedentary behaviour in the workplace}

BMJ 2018; 363 doi: https://doi.org/10.1136/bmj.k4061 (Published 10 October 2018) Cite this as: BMJ 2018;363:k4061

\section{Research}

\section{Effectiveness of the Stand More AT (SMArT) Work intervention}

\section{Cindy M Gray, senior lecturer in health behaviour change \\ Institute of Health and Wellbeing, University of Glasgow, Glasgow G12 8RS, UK}

Cindy.Gray@glasgow.ac.uk

Standing is good but moving is better

Sedentary behaviour has emerged as an important public health issue in recent years. Prolonged sedentary behaviour is associated with increased mortality, 1 cardiovascular disease, 2 type 2 diabetes, 2 colorectal cancer, 3 and poor mental health outcomes.4 Although lifestyle interventions have been shown to reduce time spent sitting, many studies are low quality and show only modest improvements in sedentary behaviour.5

One promising setting for intervening on sedentary behaviour is the workplace, which often contributes to a large proportion of people's everyday sitting. A review of interventions to reduce sitting in the workplace suggests that multicomponent interventions such as those combining sit-stand workstations with behavioural change techniques seem to hold most promise.6 High quality studies with long term follow-up are, however, lacking.

In a linked paper, Edwardson and colleagues (doi:10.1136/bmj.k3870) report the findings of a cluster randomised controlled trial showing that a workplace intervention (Stand More AT Work (SMArT Work)) was effective in reducing occupational and daily sitting time among UK hospital based office workers in both the short and the longer term (12 months).7

SMArT Work is a multilevel intervention grounded in behaviour change theories. It targets the multiple influences on employees' sitting through organisational strategies (management buy-in), environmental strategies (provision of an electric height adjustable desk or desk platform), group strategies (information session on health risks of sitting), and individual strategies, including personalised feedback, goal setting, action planning, real time self monitoring, prompts to break up sitting in the workplace, motivational messaging, and quarterly individual face-toface or telephone coaching sessions. 
The findings are important because they come from a fully powered cluster randomised trial with objective measurement of sitting time at 3,6, and 12 months, using the activPal monitor. The intervention reduced sitting and became more effective over time. By 12 months, occupational sitting in the intervention group had decreased by 83 minutes a day, compared with participants in control offices who continued with usual work practices. The intervention group also reported some improvement in musculoskeletal problems and work related outcomes, including work engagement, job performance, and occupational fatigue, compared with the control group, particularly at later time points. However, no changes in physical activity were detected.

The reported pattern of improvements in sitting over time suggests that the SMArT Work approach may produce the sustained reductions in sitting beyond 12 months that are essential for public health gain. However, baseline daily sitting in the SMArT Work trial was 9.7 hours daily. At 12 months, participants were still sitting on average more than the 6-8 hours daily, which is associated with greater risk of all cause and cardiovascular disease mortality. 8 Importantly, no change in employees' physical activity was observed; reductions in sedentary behaviour seemed to be entirely related to standing more at work. Many interventions that simply replace sitting with standing have shown clinically negligible effects on cardiovascular risk biomarkers, 9 whereas breaking up sitting with periods of even light physical activity appears to confer metabolic benefits.10 Available evidence suggests that the individual and public health benefits conferred by interventions that focus mainly on sedentary behaviour-such as SMArT Work-may be limited by their failure to increase walking or other forms of physical activity.11

The study by Edwardson and colleagues is also notable in showing improvements in self reported musculoskeletal problems and work related engagement, both of which place an economic burden on organisations owing to sick leave and reduced performance and productivity.1213 These benefits are clearly important for employers; and, once again, their emergence at later time points suggests they may be sustainable beyond 12 months.

In summary, although this is an important study that shows lasting reductions in sedentary behaviour and other work related outcomes for office based workers in UK hospitals, there are reasons for caution. Questions remain about the SMArT Work intervention's transferability beyond the National Health Service and its suitability for other types of employees, including shift workers, for whom interventions targeting sedentary behaviour are lacking.14

It is also not clear to what extent SMArT Work could be implemented beyond the research setting. One key limitation appears to be the use of the activPAL research device to provide feedback as part of the intervention. That this device requires some expert processing to obtain feedback data may be a barrier to widespread adoption. The inclusion of multiple components within the intervention, including quarterly one-to-one coaching sessions, means that although SMArT Work is effective in reducing sitting time, cost effectiveness remains unclear, particularly since physical activity did not increase. Finally, it remains unclear which elements of multicomponent interventions are most important for maximising health gains. 6 The SMArT Work study does not address this important question, which should be prioritised in future research.

\section{Footnotes}

- Research, doi: $10.1136 / \mathrm{bmj} . \mathrm{k3870}$

- Competing interests: I have read and understood the BMJ policy on declaration of interests and declare the following: none.

- Provenance and peer review: Commissioned; not peer reviewed.

\section{References}


1. Rezende LFM, Sá TH, Mielke GI, Viscondi JYK, Rey-López JP, Garcia LMT. All-cause mortality attributable to sitting time: analysis of 54 countries worldwide. Am J Prev Med2016;51:253-63. doi:10.1016/j.amepre.2016.01.022. pmid:27017420

2. Wilmot EG, Edwardson CL, Achana FA, et al. Sedentary time in adults and the association with diabetes, cardiovascular disease and death: systematic review and meta-analysis. Diabetologia2012;55:2895-905. doi:10.1007/s00125-0122677-z. pmid:22890825

3. Ma P, Yao Y, Sun W, Dai S, Zhou C. Daily sedentary time and its association with risk for colorectal cancer in adults: A dose-response meta-analysis of prospective cohort studies. Medicine (Baltimore) 2017;96:e7049. doi:10.1097/MD.0000000000007049. pmid:28562564

4. Schuch F, Vancampfort D, Firth J, et al. Physical activity and sedentary behavior in people with major depressive disorder: A systematic review and meta-analysis. J Affect Disord2017;210:139-50. doi:10.1016/j.jad.2016.10.050. pmid:28033521

5. Martin A, Fitzsimons C, Jepson R, et al. Interventions with potential to reduce sedentary time in adults: systematic review and meta-analysis. Br J Sports Med2015;49:1056-63. doi:10.1136/bjsports-2014-094524. pmid:25907181

6. Chu AHY, Ng SHX, Tan CS, Win AM, Koh D, Müller-Riemenschneider F. A systematic review and meta-analysis of workplace intervention strategies to reduce sedentary time in white-collar workers. Obes Rev2016;17:467-81. doi:10.1111/obr.12388. pmid:26990220

7. Edwardson CL, Yates T, Biddle SJH, et al. Effectiveness of the Stand More AT (SMArT) Work intervention: cluster randomised controlled trial. BMJ2018;636:k3870.

8. Patterson R, McNamara E, Tainio M, et al. Sedentary behaviour and risk of all-cause, cardiovascular and cancer mortality, and incident type 2 diabetes: a systematic review and dose response meta-analysis. Eur J Epidemio/2018;33:811-29. doi:10.1007/s10654-018-0380-1. pmid:29589226

9. Hawari NS, Al-Shayji I, Wilson J, Gill JM. Frequency of breaks in sedentary time and postprandial metabolic responses. Med Sci Sports Exerc2016;48:2495-502. doi:10.1249/MSS.0000000000001034. pmid:27387291

10. Chastin SF, Egerton T, Leask C, Stamatakis E. Meta-analysis of the relationship between breaks in sedentary behavior and cardiometabolic health. Obesity (Silver Spring)2015;23:1800-10. doi:10.1002/oby.21180. pmid:26308477

11. Stamatakis E, Gill JMR. Sitting behaviour and physical activity: two sides of the same cardiovascular health coin?Br J Sports Med2018;published online 10 Jul. bjsports-2018-099640. doi:10.1136/bjsports-2018-099640. pmid:29991572

12. Hansson EK, Hansson TH. The costs for persons sick-listed more than one month because of low back or neck problems. A two-year prospective study of Swedish patients. Eur Spine J2005;14:337-45. doi:10.1007/s00586-0040731-3. pmid:15150703

13. Schaufeli WB, Taris TW, Van Rhenen W. Workaholism, burnout, and work engagement: three of a kind or three different kinds of employee well-being?App/ Psycho/2008;57:173-203. doi:10.1111/j.1464-0597.2007.00285.x.

14. Demou E, MacLean A, Cheripelli LJ, Hunt K, Gray CM. Group-based healthy lifestyle workplace interventions for shift workers: a systematic review. Scand J Work Environ Health2018; published online 9 Sep.

doi:10.5271/sjweh.3763. pmid:30196312 